\title{
UNA PROPUESTA INTEGRAL DE EVALUACIÓN EN CIENCIAS*
}

\author{
RODRÍGUEZ BARREIRO", L. M., GUTIÉRREZ MUZQUIZ, F. A. ${ }^{2}$ y MOLLEDO CEA, J. ${ }^{3}$ \\ ${ }^{1}$ Instituto de Bachillerato Nájera (La Rioja). \\ 2 Instituto de Bachillerato Mixto núm. 15 (Zaragoza). \\ ${ }^{3}$ Instituto de Bachillerato Mixto de Tauste (Zaragoza).
}

* Un primer esbozo de este artículo aparece en el boletín interno de los CEP's de la Rioja.

\section{SUMMARY}

In the following study, we are going to deal with evaluation problems, one of the aspects in the curriculum that has undergone less changes in the last years. In order to do so, we will analyse critically the way it is done nowadays. Next, we will enunciate a series of force-ideas to propitiate the change in this field of activity. Finally, we will show the ethnographic evaluation pattern, the one we have chosen, going deeply into the methods and techniques for datacollecting more commonly used in this kind of research. Besides, we will try to explain those patterns with examples, mostly taken from our everyday practice with BUP students.

\section{INTRODUCCIÓN}

A pesar de las retóricas afirmaciones de algunos «expertos» en cuestiones educativas, la evaluación es uno de los aspectos curriculares que menos cambios ha experimentado en los últimos años. Si, como parece, existe cierto consenso a la hora de calificar de mediocre la enseñanza actual, ¿qué decir, entonces, del nivel de los procedimientos valorativos? La situación puede agravarse aún más, sobre todo en los tiempos que corren, ya que, como señala Calonghi (1978), los criterios de evaluación, pronto o tarde, consciente o inconscientemente, explícita o implicitamente se constituyen de hecho en los fines de la evaluación. En otras palabras: cualquier intento de mejora de los procesos de enseñanza y aprendizaje está condenado al fracaso - a la falta de operatividad-si no va acompañado, paralelamente, de un perfeccionamiento de los modelos y técnicas de evaluación.

En to que sigue abordaremos estos y otros problemas. Para ello, dividiremos la exposición en dos partes. En la primera, comenzaremos analizando críticamente la forma en que se realiza, hoy en día, la evaluación. Después, enunciaremos una serie de ideas fuerza que pueden servir de guía para el cambio en este campo. Por último, presentaremos el modelo de evaluación etnográfico por el que, como grupo de trabajo, nos hemos decantado. En la segunda, trataremos de mostrar algunas de las estrategias de recogida de datos-métodos y técnicas de evaluación- más usadas en la investigación etnográfica. Procuraremos, además, ilustrar dichos instrumentos con ejemplos extraídos, en su mayoría, de nuestra práctica habitual con estudiantes de BUP. Finalmente, presentaremos el esquema de evaluación que, en principio, y a la espera tanto de la negociación con los alumnos como de su operatividad, hemos puesto en marcha durante los últimos cursos.

Aunque el ámbito en el que los autores nos movemos es el de la enserianza de las Ciencias Experimentales, esta. mos seguros de que lo que aquí se va a mostrar puede ser de gran utilidad en cualquier área o nivel educativo.

\section{LA SITUACIÓN ACTUAL}

Una caracterización aproximada de la situación vigente podría ser ésta: la evaluación sólo refleja, de modo casi exclusivo, la calificación obtenida por los alumnos y alumnas en uno o varios exámenes. 
Diversas razones, de las que más tarde hablaremos, han ido relegando la evaluación a una función marginal, alejada, desde luego, de su dimensión pedagógica de ayuda al aprendizaje. La tónica habitual consiste en desarrollar una unidad de enseñanza (lección, unidad didáctica, etc.) y realizar, al final de la misma, una prueba escrita. Este proceso se repite cierto número de veces a lo largo del curso, de manera que la nota final viene a ser una especie de promedio de las calificaciones dadas con anterioridad. Es lo que, técnicamente, se conoce como evaluación sumativa (criterio).

No es de extrañar, por tanto, que, como dice Pérez Juste (1986), la evaluación sólo cumpla una función de control y de sanción social -que permite a los estudiantes promocionar o no dentro del sistema educativo-, tan mal vista por el alumno como la declaración de la renta por la mayoría de los ciudadanos.

Aparte de los problemas de indole ideológica que dicha situación plantea, hay una cuestión que nos parece capital: los exámenes son instrumentos de recogida de información cuya validez y fiabilidad suelen ser insuficientes. Como es sabido, los instrumentos de medida utilizados en educación, o los números que dichos instrumentos nos proporcionan, deben reunit ciertas características. Una de ellas es, por supuesto, que midan aquel aspecto que nos interesa, lo que se conoce como validez y, la otra, que los números asignados resulten creibles, fiables. De otro modo, y ciñéndonos a la fiabilidad como estabilidad, un examen poseerá esta cualidad si es valorado de la misma forma por distintas personas, o por la misma si se encuentra en situaciones diferentes. Una prueba tendrá validez de contenido cuando recoja una representación muestral de la población de objetos evaluables (Hemández y Sancho 1989, Escudero 1985).

Las amenazas que acechan a estos dos requisitos son muy numerosas. En cuanto al primero, las causas más frecuentes de variabilidad en las puntuaciones dadas a un mismo examen se pueden resumir así (Forns 1980): la personalidad de los evaluadores en relación con el tratamiento de la información que lleva a tratar los datos de manera distinta, bien porque la secuencia en la lectura de las pruebas cambia (la calidad de los que se llevan leídos influye en los siguientes), bien por las infornaciones previas sobre el alumno (tendencia a equiparar las notas con las anteriormente recibidas). Pero, además, la falta de fiabilidad también puede aplicarse a las realizaciones de los alumnos. Distintos alumnos pueden llegar a los mismos resultados mediante procesos cognitivos de muy distinta calidad. Se puede dar una respuesta acertada habiendo utilizado un mecanismo poco adecuado como la memorización mecánica, por no hablar de la copia, $y$, por el contrario, utilizar un mecanismo de razonamiento correcto adecuado al desarrollo cognitivo y llegar a conclusiones que el profesor considera inaceptables (Hemández y Sancho 1989).

Las investigaciones realizadas en este terreno, tanto aquí como en otros países, son numerosas. En un reciente trabajo (Jiménez 1986), la autora revela que una misma respuesta emitida por un determinado estudiante es valorađa de distinta forma por profesores diferentes. Utili- zando la pregunta y las dos respuestas que se muestran en el cuadro I con cuatro grupos de profesores, obtuvo los siguientes resultados:

\begin{tabular}{|c|c|c|c|c|}
\hline Grupo & Estudios & $\begin{array}{l}\text { N. de } \\
\text { profs. }\end{array}$ & $\begin{array}{l}\text { Respuesta A } \\
\vec{X} \text { (Val. ext.) }\end{array}$ & $\begin{array}{l}\text { Respuesta B } \\
\bar{X} \text { (Val. ext.) }\end{array}$ \\
\hline I & $\begin{array}{l}\text { Biologia } \\
\text { Medicina }\end{array}$ & 24 & $5,95(1-9)$ & $6,00(0,5-10)$ \\
\hline 2 & $\begin{array}{l}\text { Químicas } \\
\text { Matemáticas }\end{array}$ & 22 & $4,86(2-8)$ & $7,38(4-10)$ \\
\hline 3 & Biología & 30 & $6,08(3-9)$ & $5,88(3-9)$ \\
\hline 4 & Psicologia & 5 & $4,60(1-8)$ & $6,40(3-10)$ \\
\hline
\end{tabular}

Cuadro I
Pregunto
En el diario El País de 20 de octubre de 1985 apareció esta noticia:
«Un $15 \%$ de los escolares sufren ataque de piojos entre otoño y
Semana Santa. No se conocen exactamente las causas de las
recientes epidemias, ya que la higiene ha mejorado, pero todo
parece indicar que los piojos se han adaptadoalas nuevas condiciones,
resistiendo férreamente el efecto del DDT y otros insecticidas.»
Explica cómo crees que los piojos se han readaptado al efecto de
los insecticidas.
Respuesta A
Por ser un animal que tiene muchos partos sólo quedan vivos los
más fuertes, a los que no hace efecto el insecticida, y sus descen-
dientes son los que atacan ahora.
Respuesta $B$
Frente a la mayor cantidad de insecticidas, los piojos buscan la
supervivencia, y se han acostumbrado a elios; esto es lo que en
Biología se conoce como adaptación, hasta que finalmente no les
hace efecto, o sea, se han vnelto resistentes al insecticida, y las
nuevas generaciones heredaran esto y serán cada vez más resis-
tentes, ya que según las leyes de Mendel las nuevas generaciones
van evolucionando hasta ser más perfectas que las primeras.

En otra investigación (Gil 1988) se repartieron copias de un mismo ejercicio resuelto a distintos profesores, para que los corrigieran y calificaran. Aunque la prueba era siempre la misma, ésta iba acompañada đe una pequeña presentación que, en la mitad de las copias indicaba que se trataba de un «buen alumno» y, en la otra mitad, de un alumno «flojo». La nota media dada al «buen» alumno por unos 30 profesores fue un notable alto; la otorgada al «mal» alumno por otra treintena de profesores fue inferior a cinco.

En el campo de las matemáticas, podemos destacar la investigación reseñada por Marie-Claire Dauvisis (1985) en su tesis doctoral. En una experiencia de multicorrección de copias del antiguo BEPC (examen que concluía la escolaridad en los colegios franceses, hacia los 15 años), observó, con sorpresa, que las calificaciones po" dían oscilar en 11 puntos sobre un total de 20 , entre las 
notas asignadas por diferentes correctores a una misma copia. Trabajos semejantes se han realizado en nuestro país con la prueba de selectividad, obteniéndose resultados análogos.

Por lo que respecta a la validez, la mayoría de los exámenes no intenta valorar los contenidos señalados en la programación o los que están «in mente» en el profesor. Esto produce una serie de efectos no deseados, entre los que se pueden destacar los siguientes:

- En algunas áreas, como la de ciencias, hay una sobreconsideración de la aplicación (los consabidos problemas) en los exámenes.

- En otras ocasiones, para evitar un alto número de fracasos se aprecia una tendencia a saturar las pruebas de los objetivos más bajos de la taxonomía.

- El rechazo de Ios alumnos lleva consigo que no se preparen adecuadamente: se estudia para aprobar y no para saber. Así una vez superado el examen se olvida con rapidez (Pérez Juste 1986).

Pues bien, a pesar de estas y otras amenazas a la validez y a la fiabilidad, es difícil abandonar el examen como instrumento fundamental de valoración. Los motivos son diversos. Los profesores, en general, no hemos recibido la formación que nos permita tener en cuenta todos los factores que intervienen en el proceso de enseñanza y aprendizaje. Otras pruebas, distintas a los exámenes, no suelen gozar de mucha credibilidad, pues proceden de campos supuestamente «menos científicos» y, en todo caso, su utilización exigiria el aprendizaje de ciertas estrategias poco accesibles, por el momento, para la mayoría de los profesores. Si añadimos una situación tan desfavorable como el elevado número de alumnos que cada profesor tiene que evaluar, se entiende que el examen sea nuestro fundamental, y a veces único, recurso valorativo.

\section{PRINCIPIOS-GUÍA PARA UN NUEVO TIPO DE EVALUACION}

Llegados a este punto, y a partir de lo dicho hasta el momento, estamos en condiciones de rechazar sin ambages el concepto restringido de evaluación que domina en nuestro contexto: el referido a la simple calificación del alumno, de modo cuantitativo, que sirve para que éste promocione o no dentro del sistema educativo. Entre las ideas-fuerza que pueden ayudarnos a reconceptualizar la evaluación destacaremos las siguientes:

1) La evaluación ha de estar integrada en el proceso de enseñanza y aprendizaje y, por ende, debe servir para modificar aspectos relacionados con el mismo. Queda ya lejos la idea de que primero se enseña y luego se comprueba lo que se ha aprendido. Se trata, ahora, de controlar todo el proceso desde el principio hasta el final, utilizando la información obtenida para retroalimentar continuamente su desarrollo. Esto supone, entre otras cosas, que:

- La evaluación no ha de centrarse, exclusivamente, en los resultados finales, sino que debe proporcionar información, tanto al profesor como a los alumnos, sobre qué iniciativas podrán tomar ambos para modificar su trabajo y mejorar su práctica.

- La evaluación no ha de limitarse a determinar el númerode alumnos que superan los objetivos programados, sino a discernir el progreso de cada uno de ellos.

Según esto, la evaluación debe reflejar la calidad -más que la cantidad- del aprendizaje, apoyándose en la observación de los avances conseguidos por cada estudiante (Driver 1987). Por consiguiente, no bastará con averiguar si se ha producido la comprensión de los distintos temas, sino que habrá que recabar informaciones de otra índole: trabajo en la clase, capacidad para analizar y tratar datos, aplicación de los conocimientos, actitudes hacia la asignatura, aptitud para las tareas cooperativas, etc.

2) El alumno debe participar en la actividad evaluatoria, al menos, en dos sentidos:

- Ha de poder percibir los avances en su propio aprendizaje. Para ello hay que darte, en la secuencia de enseñanza, oportunidad de reconocer sus propias ideas -evaluación inicial-y de observar cómo cambian y que progresos ha realizado -evaluación formativa-.

- Ha de participar en las tareas de evaluación de la marcha general del curso, de manera que sus aportaciones sean tenidas en cuenta e influyan en el desarrollo del mismo.

3) Una evaluación integrada en el proceso de enseñanza y aprendizaje, y concebida como un instrumento de ayuda pedagógica, nos obliga a considerar, además de la obligada (?) valoración final -evaluación sumativa-, otros momentos en los que ejercer la evaluación. Nos referimos a las denominadas evaluación inicial y evaluación formativa.

La evaluación inicial es la que pretende detectar el estado de conocimientos que poseen los estudiantes cuando comienzan un tema. En palabras de Coll (1987), persigue evaluar los esquemas de conocimientos pertinentes para el nuevo material de aprendizaje. Recuérdese que, según la perspectiva constructivista, se aprende a partir de lo que ya se sabe, y que el aprendizaje se produce si el alumno es consciente de sus conocimientos previos. Esta evolución no debe limitarse a las pruebas escritas tradicionales -puede que incluso éstas sean contraproducentes-; es preferible, por el contrario, utilizar técnicas variadas, desde la realización por los alumnos de mapas conceptuales hasta los debates sobre una situación problemática, pasando por los consabidos cuestionarios, etc. Su objetivo último residiría, pues, en recoger datos sobre las ideas previas y los errores conceptuales de los alumnos, información básica para el profesor a la hora de programar y para el alumno de cara al aprendizaje. 
La evaluación formativa debería ser la base del proceso evaluador. Su finalidad no es la de controlar y puntuar a los estudiantes sino la de ayudarles pedagógicamente a progresar en los conocimientos, en la instrucción que se les imparte. Implica para el profesor -y para el alumno- una tarea de ajuste constante para irse ade. cuando al desarrollo de los alumnos y para establecer nuevas pautas de actuación en relación con los resultados obtenidos en el aprendizaje (Hemández y Sancho 1989).

Es un hecho que muchos profesores llevan a cabo este tipo de práctica, aunque de forma generalmente intuitiva $y$, en ocasiones, sin ser siquiera conscientes de ello. Sin embargo, nos parece necesario que se haga de un modo más riguroso, recurriendo a algún modelo teórico y a las numerosas técnicas disponibles y adecuadas para facilitar el seguimiento de los avances y los bloqueos en el proceso de enseñanza y aprendizaje. Entre estas últimas, se pueden citar, de momento, las tablas de observación y las hojas de seguimiento.

La evaluación sumativa -final- debe reunir ciertos requisitos. Por ejemplo, es preciso definir con claridad el campo a medir, así como el patrón (¿cuando se adjudica un cinco?). Ambos aspectos deberían no sólo ser hechos públicos, sino discutidos con los propios alumnos. También sería conveniente emplear una mayor variedad de ins. trumentos de evaluación, cuidando, eso sí, su fiablidad y validez.

Este esquema podría concluirse con ta elaboración y aplicación de un cuestionario - u otra técnica pertinenteen el que los alumnos pudieran evaluar el trabajo realizado.

4) Las tres modalidades de evaluación que acabamos de revisar han de aplicarse no sólo al dominio cognitivo, como acostumbra a hacerse hoy en día, sino, además, a los dominios psicomotor y afectivo. Aunque la valoración en este último ámbito todavía se mira con algunas reticencias, es obvio, como señala Escudero (1985), que los intereses, los sentimientos, las actitudes y los valores ante las distintas asignaturas de los alumnos deben estar en el punto de mira permanente del profesor. Si se desea tener una panorámica de las tendencias actuales en la evaluación de actitudes, una referencia interesante puede ser el fascículo 11 de las guías curriculares del proyecto \$SCR (1987).

En el dominio psicomotor, esto es, el referido a las destrezas y habilidades en contextos prácticos, la bibliografía reciente es bastante extensa. De hecho, es posible que ésta sea una de las líneas de investigación que mayor desarrollo ha experimentado en los últimos años (Alberts et al. 1986).

5) La evaluación no debe centrarse exclusivamente en el alumno. Habrá de abarcar otros elementos cuya consideración es determinante a la hora de alcanzar el «éxito» en nuestro trabajo. A modo de ejemplo, y siguiendo a Escudero (1985), sería conveniente evaluar:

- Al profesor, su estilo personal, etc.
- EI método o enfoque de enseñanza que utilice: aprendizaje como investigación, clase magistral. etc.

- El ambiente que haya en el aula, las interacciones que en ella se produzcan (profesor-alumno, alumno-alumno, etc.).

- La propia programación y los materiales curriculares que se empleen (unidades didácticas, técnicas de evaluación...).

6) Los puntos anteriores exigen, por último, la utilización de técnicas variadas y flexibles. Tanto el grado de exigencia respecto a su frabilidad y validez como el coste en términos de dificultad y tiempo de elaboración y aplicación deben ser sopesados por el profesor en función de sus intereses concretos.

Parece evidente, por otra parte, la necesidad de que este trabajo sea llevado adelante por el equipo de profesores, ya se trate de un seminario o de un departamento. Los miembros de estos grupos deberían establecer criterios comunes de evaluación, respetando, de algún modo, la autonomía individual, con el propósito de que las relaciones entre cada profesor y sus alumnos no se vean comprometidas.

\section{HACIA UNNUEVOMODELODEEVALUACXÓN}

Plasmar en los hechos Ias ideas que se acaban de mencionar conlleva un nuevo significado del concepto de evaluación; evaluar equivale, entonces, a recapacitar sobre el proceso global de enseñanza y aprendizaje, es decir, a investigar. Es posible que a alguros compañeros les parezca excesivo tener que desempeñar el papel de profesor investigador; sin embargo, como sef̃ala Erickson (1989) para que la enseñanza primaria y secundaria alcance su mayoría de edad como profesión-para que el docente no continúe siendo institucionalmente infantilizado- los profesores deben asumir la responsabilidad adulta de investigar su propia práctica en forma sistemática y critica mediante los métodos apropiados.

Nuestro próximo paso habrá de consistir, por consiguiente, en seleccionar un modelo de investigación que, siendo coherente con las ideas-fuerza anteriores, permita ponerlas en acción. Y hablamos de modelo teórico, ya que una técnica de investigación no constituye un método de investigación. Los datos obtenidos con aquélla nunca se explican por sí mismos, sino que su interpretación está condicionada por los supuestos teóricos del investigador - del profesor, en este caso-.

Existen, en esencia, dos modelos de evaluación: el ex. perimental, que emplea sobre todo instrumentos cuantitativos, y el etnográfico o interpretativo, caracterizado por recurrir preferentemente a las técnicas cualitativas. El primero se basa en el control inicial de las variables implicadas en el estudio -por ejemplo, conocimientos previos, inteligencia, etc.-, la aplicación de las medidas 
correspondientes -verbigracia, un nuevo modo de enseñar, un plan para el desarrollo de la inteligencia, etc. - y el análisis final que permita comprobar qué modificaciones ha producido la actividad en cuestión (Kerlinger 1975). Siguiendo a Santos (1988), este planteamiento nos impide analizar la evolución del trabajo, el funcionamiento de la caja negra donde se desarrollan los procesos, la dinámica interna que se establece entre los protagonistas de la actividad y las intenciones, los motivos y las valoraciones de quienes están llevando a cabo la tarea.

Es por ello que hemos preferido optar por el modelo etnográfico. Para caracterizarlo puede resultar muy útil comparario con el experimental -más propio de las ciencias físico-químicas-. La principal diferencia radica, probablemente, en los respectivos supuestos acerca de lanaturaleza de la causalidad en las relaciones humanas (Erickson 1989). En cuanto a otros aspectos, como el papel de la teoría, de la evidencia empírica, de las categorías de análisis y de la objetividad, la etnografía es:

- Inductiva, ya que trata de descubrir una teoría que explique sus datos.

- Generativa: pues procura descubrir conceptos y proposiciones a partir de varias fuentes de datos.

- Constructiva: en tanto que pretende descubrir unidades de análisis a partir de la observación y de la descripción.

- Subjetiva, ya que incorpora los datos subjetivos (del profesor investigador y de sus alumnos).

Por el contrario, el modelo experimental se situaría, en las mismas dimensiones, más cerca de los polos deductivo, verificativo, enumerativo y objetivo; o lo que es lo mismo: su fin prioritario consiste en contrastar una teoría, determinando en qué medida se cumple una proposición, para lo que recurre a unidades de análisis elaboradas previamente y en las que no considera lo subjetivo (Goetz y Lecompte 1988).

La naturaleza de los fenómenos que ocurren en el aula pensamos que debe ser determinante a la hora de inclinarse por el modelo etnográfico, lo que no quiere decir, ni mucho menos, que se rechacen algunos instrumentos cuantitativos, más propios del otro enfoque. No conviene olvidar, además, que etnógrafos educativos de gran experiencia, como Hammersley (1990), han llegado a plantear la necesidad de introducir algunas modificaciones en el modo en que la etnografía se aplica en las escuelas; principalmente, en dos campos. Primero, la selección de casos para la investigación debe proporcionar la modificación de las variables teóricas y, al menos, un control parcial de las variables extrañas relevantes. Segundo, la operacionalización de los conceptos ha de hacerse con más rigor. En otras palabras, se trata de un acercamiento a ciertas características del modelo experimental.

Pero ya es hora de que nos centremos en la segunda parte del artículo. A pesar de la «dureza» de los últimos párrafos, éste será el momento de comprobar lo «sencillo" que puede resultar poner en marcha el nuevo modelo de evaluación que acabamos de compendiar.

\section{ESTRATEGIAS DE RECOGIDA DE DATOS: TIPOS Y EJEMPLOS}

Con el fin de conceptualizar correctamente los distintos métodos, recurriremos para su presentación a una de las clasificaciones más extendida: la que los divide en in. teractivos y no interactivos (Goetz y Lecompte 1988), bien entendido que, a la hora de la verdad, ambos tipos no sólo no se excluyen entre sí, sino que suelen utilizarse de modo complementario. De hecho, algunas de las estrategias recopiladas dentro del segundo grupo podrían, sin lugar a dudas, ser incluidas en el primero.

Por otra parte, debemos recordar que estos instrumentos permiten, en primer término, realizar la evaluación en los tres momentos clave: inicial, formativo y sumativo; en segundo, aplicarla en los tres dominios clásicos: cognitivo, psicomotor y afectivo; $y$, por ultimo, valorar no sólo a los estudiantes sino al resto de los aspectos ya reseñados: materiales curriculares, programas, profesores, procesos de instrucción, etc. Como es natural, algunos de ellos cumplen mejor que otros determinadas funciones, por lo que, en tales casos, así lo haremos notar expresamente. Un par de buenos resúmenes de sus ventajas e inconvenientes, así, como del campo de aplicación, pueden encontrarse en las obras de Walker (1939) y Wittrock (1989).

Dado el reducido espacio disponible, nos limitaremos a dar una rápida panorámica de los distintos métodos. El lector interesado podrá profundizar en el terna sin más que recurrir a las diversas referencias bibliográficas que acompañan al artículo.

\section{Métodos interactivos}

Están basados, como su nombre indica, en la interacción personal de los participantes en el estudio (en el caso que nos ocupa, alumnos y profesores). A través de ésta el investigador puede obtener, con más facilidad que me. diante otros métodos menos intrusivos, la información relevante para las cuestiones que se haya planteado. Las dos estrategias más importantes son:

- La observación participante. Es el instrumento etnográfico por excelencia. En ella, y en sentido estricto, el investigador pasa todo el tiempo posible con los individuos que estudia; refleja sus interacciones y actividades en notas de campo que toma en el momento o inmediatamente después de producirse los fenómenos.

Sin embargo, desde la perspectiva de un docente en el aula, creemos que su papel debería acercarse más al de un participante excepcionalmente observador, que podría recoger sus notas en lo que se ha dado en llamar diario del profesor. Éste puede ser, sin duđa, un utensilio valiosísimo para el análisis crítico de los procesos de aprendizaje -por ejemplo, en la detección de las dificultades y bloqueos que nuestros alumnos encuentran a la hora de aprender- y para ayudar al profesor a tomar decisiones que le permitan mejorar su trabajo. 
Cuadro II

\author{
Curso: $2^{\circ}$ de BUP \\ Fecha: 14-2-90. \\ Diario: Sin ayuda de magnetofono \\ * Realizado una hora después
}

A 19. Dado que esta activicad está encaminada a la detección de las posibies ideas previas de los alumnos, pido que la lean detenidamente. Sin debate en grupo, pregunto de forma abierta: ¿qué pensáis acerca de la fuerza que debe hacer la persona de la figura si desea seguir moviéndose?

Iñaki: «Yo creo que no necesita ningura. Se seguirá moviendo.»

Cristina: «Cómo que no?, sí que necesita hacer fuerza, ya que si no se irá parando poco a poco."

Cristina, ¿por qué supones que se iría parando progresivamente?

Cristina: «Porque la fuerza que lieva al bajar se irá agotando hasta que se pare.»

Profesor: «Qué os parece al resto de la clase?».

Curiosamente, 19 alumnos apoyan la primera propuesta y 6 la segunda.

Ahora, repito la pregunta en un nuevo contexto:

Profesor: «Imaginaos una bolita que rueda sobre un plano horizontal muy pulimentado después de haberla puesto en movimiento con un pequeño impulso; ¿terninará deteniéndose o seguirá su movimiento indefinidamente?»

Todos los alumnos mantienen sus posiciones iniciales.

Iñaki insiste en que no habría motivo para que se detuviera: «... bueno, se pararía si rozase con la nieve.»

Varios alumnos: « $\mathrm{Si}$, sí; si rozase se llegaría a parar.»

Cristina y su grapo de inconcicionales aseguran que «la fuerza con la que baja se acabatá y lerminará parándose. Eso pasa siempre, en todos los casos en que se lanza un objeto.»

Iñaki: «Pero si no roza, yo creo que no hay motivo.»

Victoria: "indefinidamente!, eso es demasiado."

Dejamos por el momento la puesta en común. Pido a todos los grupos que si tienen que hacer alguna anotación en sus cuadernos ahora pueden dedicar unos instantes.

Como viene siendo costumbre en las actividades de detección de idess previas de los alumnos, voy a procurar condicionar lo menos posible las respuestas q̨ue se emitan. Planteo mi actuación a modo de conversación con cada uno de los alumnos que intervienen.

Será conveniente analizar la eficacia de las pregun tas abiertas en este tipo de cuestiones. Me propongo extraer en estas dos primeras activiofades una idea del número de alumnos que comparten cada una de las alternativas presentadas.

Durante la clase de hoy todos los grupos se han mostrado especialmente activos en la puesta en común. ElG S se muestra muy bullicioso. Tengo que llamarles varias veces la atención. Me han asegura do que están trabajando sobre la actividad.

Habrá que analizar la conveniencia o no de plantear nuevos casos particulares de una actividaỏ ya planificada, en particular cuando se anda escaso de tiempo

Cristina me pregunla de nuevo por que no doy la solución, «pero es una cosa u atra, venga dím. Les recuerdo de nuevo la intencion que se persigue con este tipo de actividades: «analizando las siguientes cuestiones trataremos de encontrat entre todos las ideas aceptadas cientificamente».
Porlán (1987) señala tres estilos diferentes en la elaboración del diario, según el tipo de observaciones que se realicen:

\section{- Centrado en las emociones.}

- Centrado en las actividades de aprendizaje.

- Centrado en la dinámica social de la clase.

En el cuadro II se muestra un fragmento de un diario que bien podría enclavarse en el segundo grupo. La actividad descrita corresponde a la unidad didáctica «Fuerzas y Movimiento». Con ella se pretendía averiguar las ideas previas de los alumnos sobre el concepto de fuerza.

Nuestra experiencia nos indica que confeccionar un diario de clase es una tarea costosa y que, sobre todo, exige tiempo. Este inconveniente, agravado por el excesivo número de horas lectivas con el que contamos, no impide, sin embargo, que insistamos en recomendarlo. Una manera de superar dicho obstáculo residirfa en «enfocar» exclusivamente la atención en aquellas cuestiones por las que estemos más interesados durante un período determinado, verbigracia, a lo largo de una unidad didáctica o en el transcurso de una actividad práctica en el laboratorio.

- La entrevista. Constituye un excelente medio auxiliar, ya que proporciona información sobre numerosas habilidades: cognitivas, afectivas... Para nosotros, la entrevista cobra cada día mayor importancia, ya que permite completar la información recabada mediante la observación participante, así como la recogida con otros instrumentos menos potentes: cuestionarios y pruebas escritas, en general. Tal contraste, además, posibilita que se puedan Ilevar a cabo las triangulaciones que aseguren la validez de los datos.

Cuando el docente se plantea, con rigor, el análisis de las dificuliades que sus alumnos encuentran durante el aprendizaje, la entrevista se convierte en una de las mejores herramientas disponibles. $Y$, aunque de nuevo se requiere cierto tiempo para una correcta realización de la misma, también ahora es posible solventar este inconveniente. Basta con entrevistar tan sólo a una pequeña muestra, representativa respecto de la cuestión planteada, del total de la población estudiada. 
En el cuadro III se presenta un extracto de una entrevista, realizada con una muestra de alumnos de $1^{\circ}$ de BUP, al cabo de un mes de finalizada la unidad didáctica «Las cadenas alimentarias, elementos básicos para el estudio de un ecosistema».

Cuadro thI

\section{P. ¿Qué significa cadena alimentaria?}

A. Un conjunto de seres que se van alimentando unos a otros... que luego se alimentan entre todos, va sigutendo unos a otros.

P. Sí, ¿la cadena de alimentación es lineal?, quiero decir, ¿siempre uno come a otro que...? (interrumpe).

A. No, puede comer a varios.

P. O sea, hablar de cadenas alimentarias igual no es muy correcto. Tú, ¿qué palabra emplearías?

\section{A. Red. Red alimentaria.}

P. Díme algunos nombres de individuos de la cadena alimentaria que ocupan siempre el mismo lugar, que, aunque no coman at mismo individuo, sí, a los que tienen... (interrumpe).

A. Primero eran los vegetales, por ejemplo la Spirogyra, que luego se comía el Rotífero, era comida, vamos, por el Rotífero; luego, pues la Pulga de agua; los Rotíferos podían comerse a otros Rotíferos.

P. Y si no me dijeras nombres concretos de animales, ¿que nombre en general vale para los escalones que has nombrado en primero, segundo y tercer lugar?

A. Los productos eran los vegetales; luego los consumidores, que había primarios y secundarios.

P. ¿Qué características durías túí que diferencian a un animal de un vegetal?

A. Que los animales se suelen mover. Y el color, sobre todo el color: los vegetales tienen cloroplastos.

P. ¿Los animales no? ¿Nunca?

A. No.

P. ¿Qué tienen los cloroplastos de especial?

A. La clorofila.

P. ¿Y pasa que sirve?

A. Para la alimentacion. A traves de la fotosíntesis los vegetales obtienen su clorofila. La ciorofila interviene en la fotosíntesis.

\section{Métodos no interactivos}

Un etnógrafo incluiría en este grupo aquellos métodos en los que se desarrolla una interacción escasa o nula con los participantes. Teniendo en cuenta la realidad del aula, nosotros situaremos aquí todos los procedimientos en los que la relación personal con los estudiantes sea menor. Parece difícil, salvo contadísimas y antediluvianas excepciones, que un profesor no tenga algún tipo de relación con sus alumnos. También incluiremos en este apartado los instrumentos que cualquier profesor puede emplear para observar la labor de otro compañero.

- Observación no participante. Consiste en contemplar lo que está aconteciendo y registrar los hechos sobre el terreno. Los tipos más usados son:

- Crónicas de flujos de comportamiento. Requieren descripciones exactas de lo que los participantes dicen o hacen. Pueden ser abiertas o cerradas, según el grado con que el profesor haya precisado sus propios intereses.

Pertenecen a este subgrupo los mapas y plantillas de observación. En el cuadro IV se muestra un mapa de una clase de $3^{\circ}$ de BUP, basado en la plantilla o tabla de observación que figura en el esquema de evaluación que proponemos al final del artículo. En ésta se señalan, asimismo, los criterios de evaluación. Mapas similares pueden construirse para observar el trabajo de laboratorio. La tabla correspondiente figura, también, en el esquema final.

La mayor ventaja de estos instrumentos, frente a los interactivos, reside en la rapidez y sencillez con las que se registran los datos.

- Protocolos de análisis de interacción. Éstos van desde los sociogramas informales, elaborados sobre el terreno por el observador, hasta sistemas estandarizados de clasificación de los comportamientos, como el de Flanders (1977). Su objetivo radica en anotar las formas en que interactúan los participantes.

No obstante, existe la posibilidad de usar, con ese fin, los mapas de observación. Son mucho más asequibles y únicamente requieren que se establezca algún sistema de símbolos para agilizar el registro (Sancho 1988).

- Medios audiovisuaies. Nos referimos a la grabación de las clases, total o parcialmente, tanto en cassette comoen vídeo. Ambas técnicas proporcionan una abundante y fiable información, por lo que su uso es cada vez mayor en la investigación educativa. Su empleo habitual conlleva un mayor coste económico, a la vez que precisa un mayor tiempo (vaciado, interpretación, etc.) que otros instrumentos. Sin embargo, su audición o visionado por parte de los alumnos suele producir un «feedback» altamente positivo. En particular, les permite tomar conciencia de la forma en que se desenvuelve su trabajo $y$, en consecuencia, les ofrece la posibilidad de mejorarlo.

- Recogida de documentos. Además de hablar e interactuar, los discentes y los docentes fabrican y utilizan cosas. Los documentos resultantes -cuaderno de clase, guiones preparatorios, etc.- se convierten en datos que indican las sensaciones, experiencias y conocimientos de las personas. 


\section{Cuadro IV}

Curso: $3^{9} \mathrm{~A}$, núm. 35

Fecha: $5,6,7$ y 8 de noviembre.
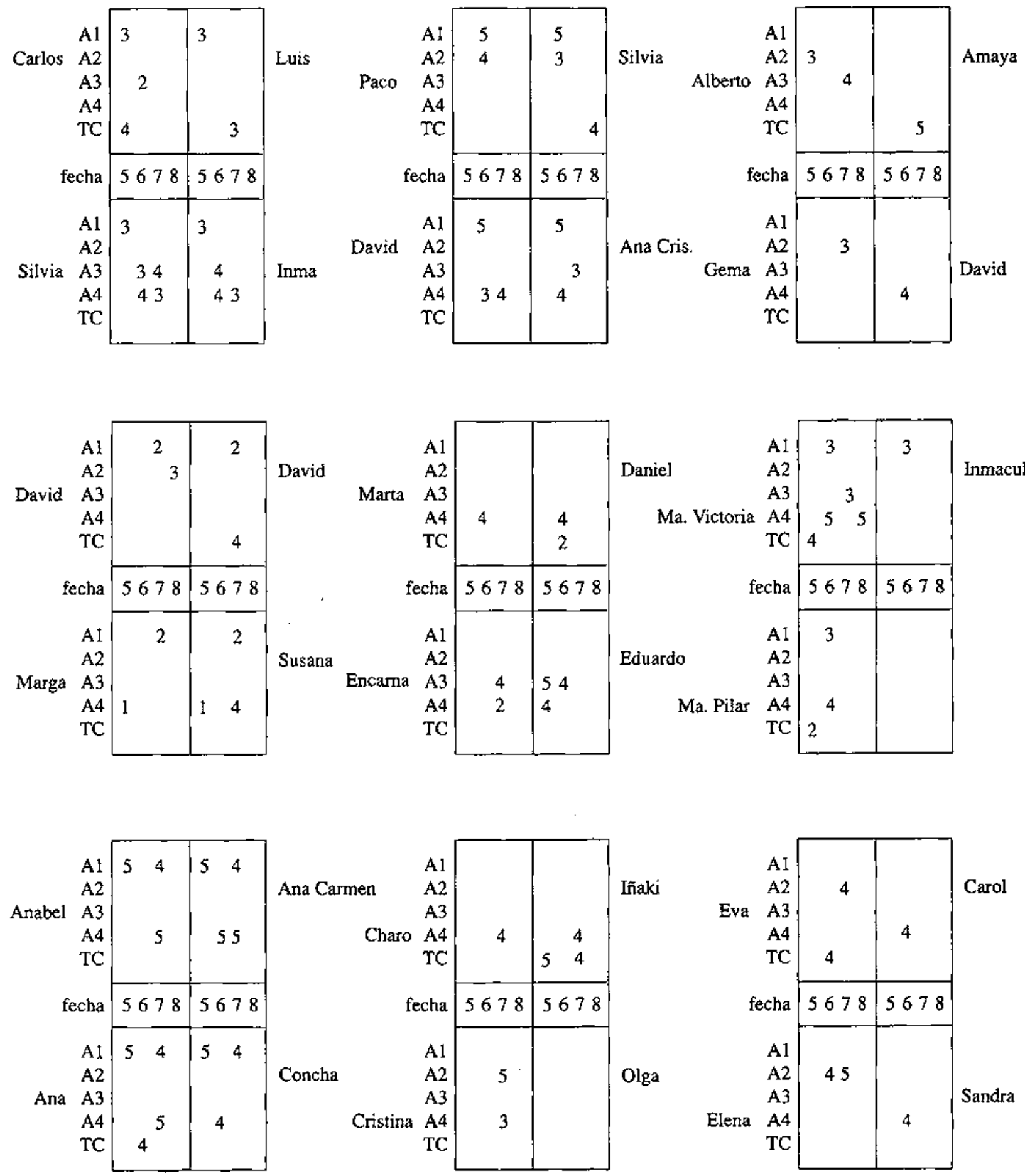

- Cuaderno del alumno. Para nosotros, y dado el enfoque de enseñanza que utilizamos, constituye una de las herramientas más importantes. En él, el alumno recoge, para cada una de las actividades, sus opiniones y las derivadas de la puesta en común, con lo que poco a poco el cuaderno se convierte en su auténtico y personal «libro de texto». En el esquema final se muestran las categorías empleadas para su corrección. 
- Pruebas de lápiz y papel: Cuestionarios, exámenes ... Habitualmente, empleamos dos tipos de cuestionarios: uno, para conocer las ideas del alumnado antes de iniciar el proceso de instruccion; y otro, para comprobar en qué medida aquéllas han evolucionado durante el mismo. En el primer caso, el profesor acostumbra a elaborar un resumen que comunica (mediante transparencias u hojas fotocopiadas) a los alumnos, con el objeto de que conozcan la diversidad de opiniones de la clase acerca del concepto tratado. El segundo tipo puede resultar adecuado para disponer rápidamente de todas las respuestas de la clase. En ambos casos, dependiendo del contexto, el profesor decide si las respuestas han de emitirse individualmente o en grupo.

Ya indicamos en la primera parte del artículo la necesidad dc especificar, en este tipo de pruebas, el campo a medir y el patrón. Pero todavía es posible mejorarlas en mayor o menor medida. Para ello, se recomienda especificar qué objetivos y en qué proporción han de incluirse en cada una de ellas. En nuestra área, y en cuanto a la evaluación del conocimiento de hechos, conceptos y teorías científicas, pueđe resultar útil la categorización hecha por Kempa (1986), basada, a su vez, en la más conocida y clásica de Bloom.

Según Kempa, cabría hablar de cuatro niveles de objetivos:

Nivel 1: Conocimiento y recuerdo de hechos, hipótesis, teorias, conceptos, terminologia y convenciones cientificas.
Nivel 2: Comprensión de los conocimientos científicos y sus relaciones manifestadas en la capacidad de los estudiantes para explicar e interpretar la información presentada y para expresarla en diferentes formas.

Nivel 3: La aplicación del conocimiento cientifico a situaciones nuevas. Implica que el alumno es capaz de seleccionar de sus conocimientos anteriores aquéllos que son interesantes para resolver una nueva situación.

Nivel 4: Análisis, síntesis y evaluación de la información científica que implica la descomposición en sus partes constituyentes y la reorganización en una nueva estruc. tura. Adicionalmente la información puede ser evaluada en términos de su validez y asunciones y consecuencias subyacentes.

Hemos de reconocer que, por ahora, todavía estamos empezando a tener en cuenta esta clasificación. Como acertadamente señalan Gutiérrez y otros (1990), la mayor dificultad estriba en evaluar tales categorías por separado, siempre que el aprendizaje sea significativo y no puramente memorístico. No obstante, unos contenidos (hechos, conceptos, principios) se prestan más que otros al desarrollo de objetivos de uno u otro nivel. El profesor debe tener en cuenta este hecho. En el cuadro V se muestra una tabla de doble entrada utilizada para representar las especificaciones de un test (de opción múltiple) correspondiente a la unidad didáctica «La estructura de la materia” de $2^{2}$ de BUP.

Cuadro V

Áreas principales de estudio.

\begin{tabular}{|c|c|c|c|c|c|c|c|c|}
\hline HABILIDADES & $\begin{array}{c}\text { Identificación } \\
\text { de sustancias } \\
\text { puras }\end{array}$ & $\begin{array}{c}\text { Obtencion de } \\
\text { sustancias } \\
\text { puras }\end{array}$ & $\begin{array}{c}\text { Concepto dc } \\
\text { proceso } \\
\text { fisico }\end{array}$ & $\begin{array}{c}\text { Elementos } \\
y \\
\text { compuestos }\end{array}$ & $\begin{array}{l}\text { Concepto } \\
\text { de proxeso } \\
\text { quimico }\end{array}$ & $\begin{array}{c}\text { Leyes } \\
\text { ponderales } \\
\text { de ta químic }\end{array}$ & $\begin{array}{c}\text { Naturaleza } \\
\text { atornica de la } \\
\text { materia }\end{array}$ & $\begin{array}{l}\text { PORCENTAII: } \\
\text { APROXMAMAOO } \\
\text { OEPUNTOS }\end{array}$ \\
\hline $\begin{array}{l}\text { Conocimiento: } \\
\text { habilidades para } \\
\text { recordat }\end{array}$ & & & & & & & & 40 \\
\hline $\begin{array}{l}\text { Comprension: } \\
\text { hamilidadedes para tra- } \\
\text { ducire interpretar } \\
\text { infortmación }\end{array}$ & & & & & & & & 30 \\
\hline $\begin{array}{l}\text { Aplicacion: habilida } \\
\text { des para aplicat los } \\
\text { conocimientos a } \\
\text { nuevas } \\
\text { situaciones }\end{array}$ & & & & & & & & 20 \\
\hline $\begin{array}{l}\text { Análisisfevaluación: } \\
\text { habibiladed para } \\
\text { analizar y evaluar } \\
\text { informaticiones } \\
\text { y datos }\end{array}$ & & & & & & & & 10 \\
\hline $\begin{array}{l}\text { PORCENTANE } \\
\text { APROXIMADO } \\
\text { DE PUNTUACION }\end{array}$ & 10 & 10 & 10 & 15 & 20 & is & 20 & 100 \\
\hline
\end{tabular}


Procedimientos semejantes se han desarrollado en la evaluación de los trabajos o habilidades prácticas. Christofi (1988) recoge algunos de ellos en su breve y clara obra sobre la evaluación en ciencias.

- Diferencial semántico. Se trata de un instrumento de muy sencilla construcción y aplicación, cuya funcionalidad en la evaluación de actitudes está plenamente demostrada (Kerlinger 1975). Para construir un diferencial semántico, en palabras de Gutiérrez y otros (1990), hay que, a) establecer pares de adjetivos que indiquen situaciones opuestas respecto a los diversos aspectos del constructo que se quiere estudiar; y b) pedir al alumno que se sitúe en una escala que suele ser de 1 a 7 según sea su postura frente a cada par de adjetivos.

En el cuadro VI se recoge un fragmento de un diferencial semántico que incluía otros cinco conceptos. Los pares de adjetivos eran siempre los mismos. Para la interpretación de los datos obtenidos se recomienda leer el capítulo 23 de Kerlinger (1975).

Las clases de ciencias son:

Buenas

Desagradables

Interesantes

Útiles

Aburridas

Fáciles

Innecesarias

Desorganizadas

Costosas

Pasivas

Eficaces

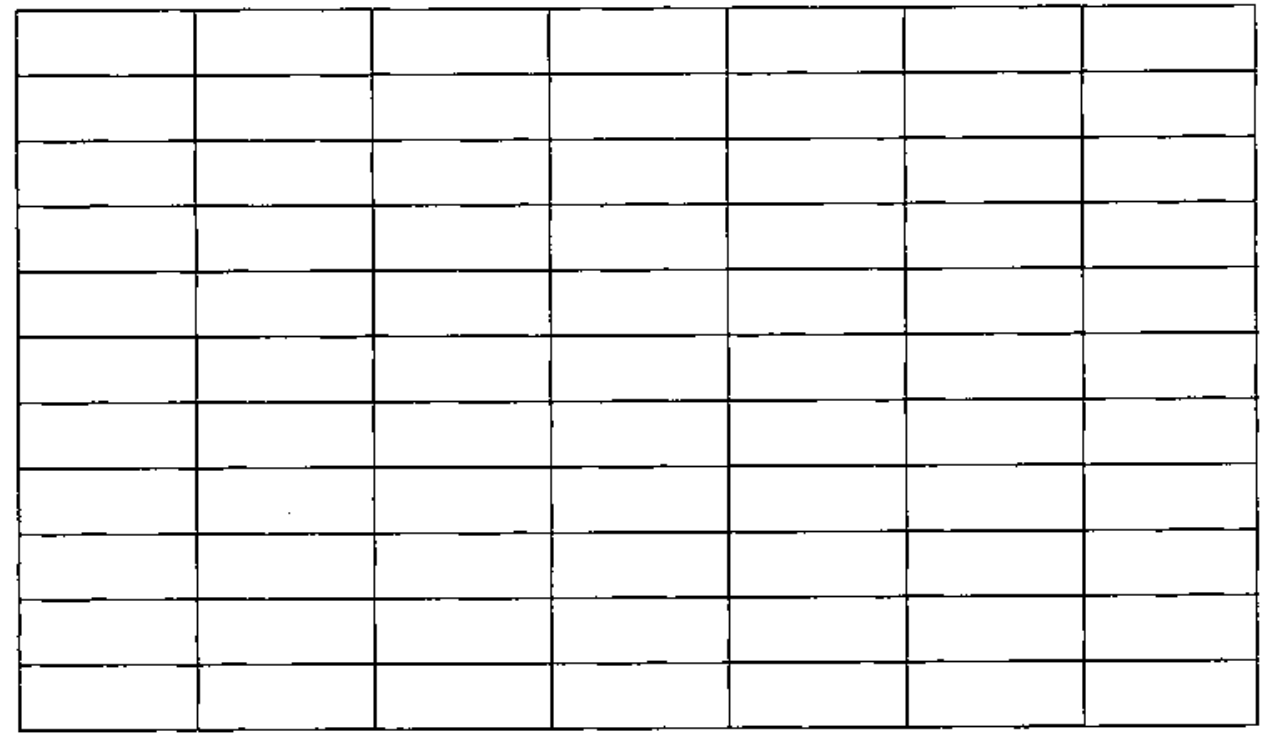

Malas

Agradables

No interesantes

Inútiles

Divertidas

Difíciles

Necesarias

Organizadas

No costosas

Activas

Ineficaces

Las prácticas de laboratorio son:

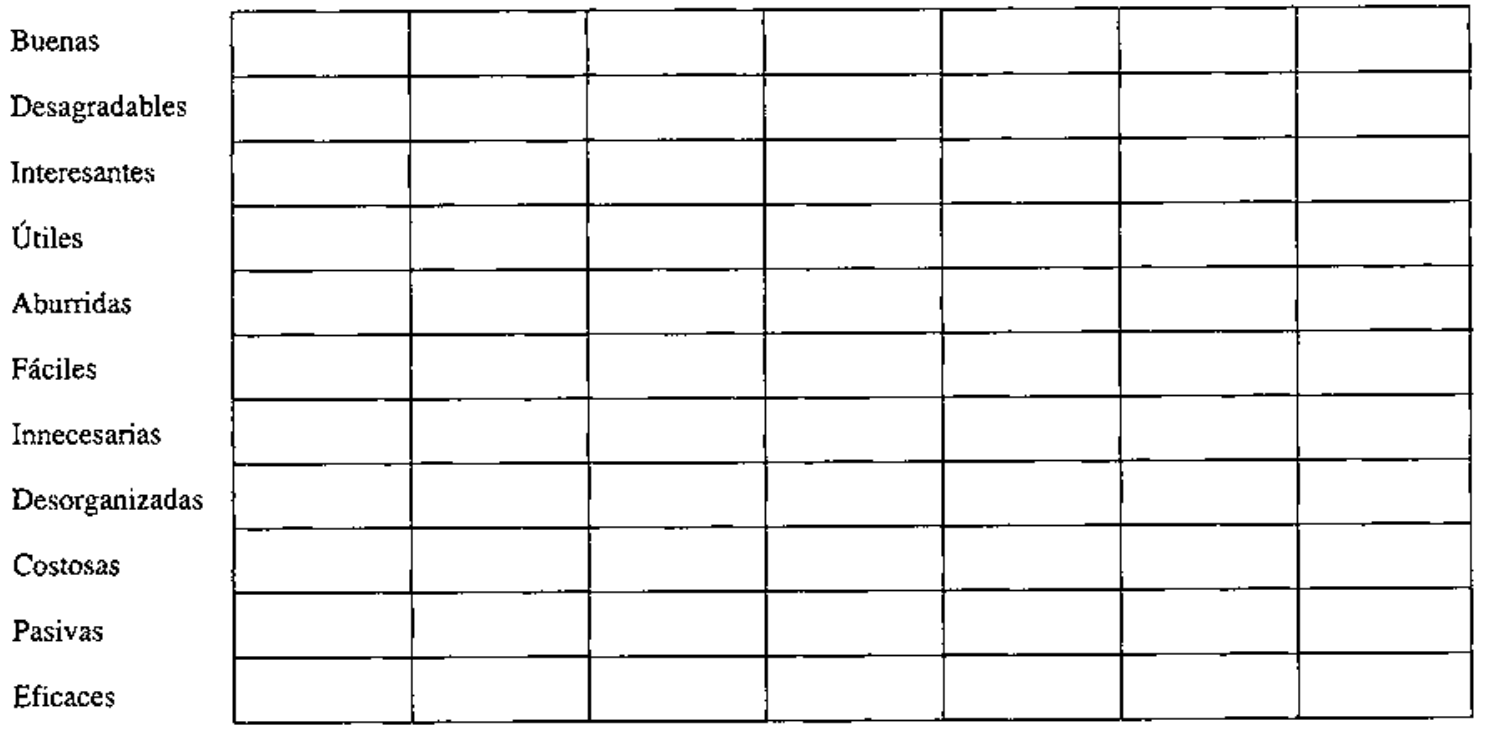

Malas

Agradables

No interesantes

Inútiles

Divertidas

Dificiles

Necesarias

Organizadas

No costosas

Activas

Ineficaces 
- Encuestas. Constituyen un medio rápido y, si están bien diseñadas, eficaz para recabar información de diversa índole. Pueden pasarse al acabar una unidad didáctica (San Valero 1988) o al finalizar el curso. La única diferencia estriba, claro está, en el tamaño y complejidad de las mismas. En el transcurso de los últimos años hemos ido perfilando una encuesta que contiene unos 90 ítems, distribuidos en los siguientes apartados:

* Valoración de los programas de actividades.

* Valoración del método de enseñanza y aprendizaje.

* Valoración del proceso de instrucción.

* Valoración del profesor.

* Valoración de los contenidos.

* Valoración del método de evaluación.

* Sugerencias.

En el cuadro VII se muestra un fragmento de elia, con las correspondientes respuestas dadas por alumnos de $2^{\circ}$ de BUP una vez terminado el curso.

\section{Cuadro VII}

Valoración del proceso de instruccion.

Califica de 1 a 5 cada una de las siguientes cuestiones referidas al método de instructivo utilizado este curso:

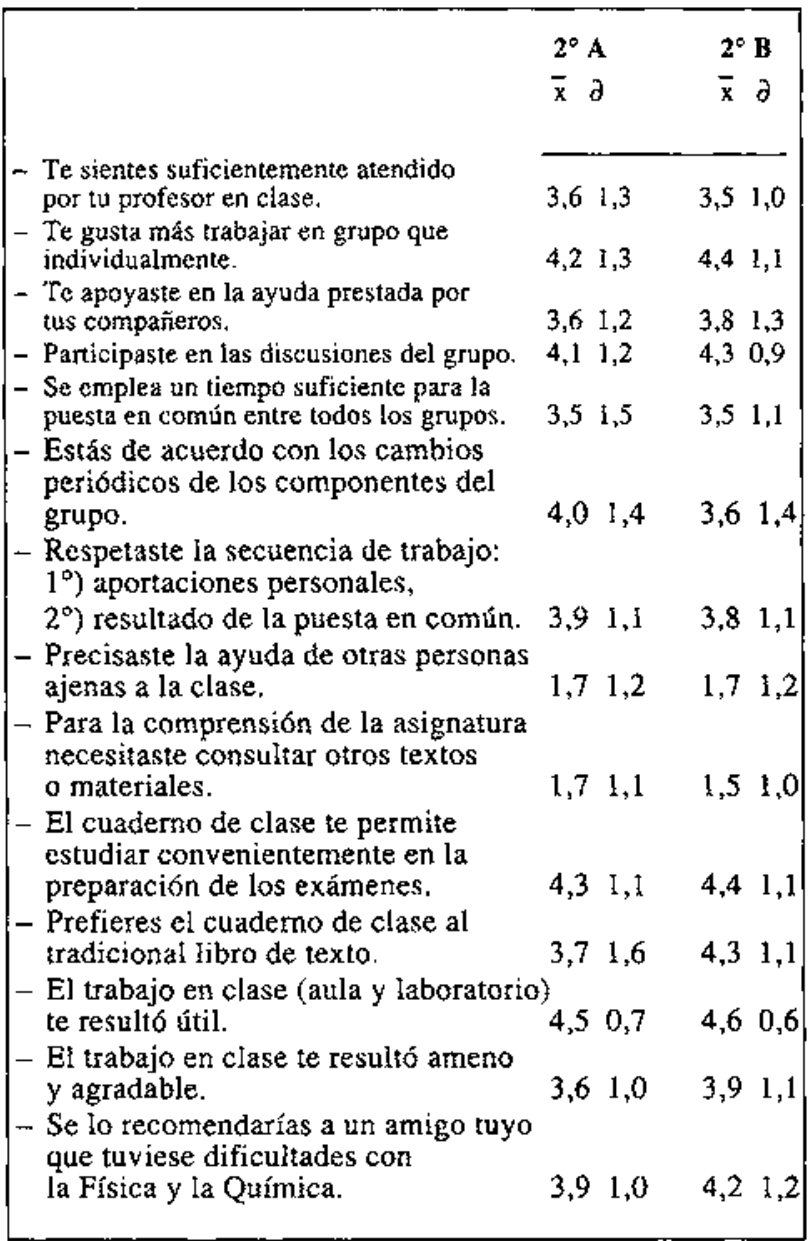

- Informes de grupo. Se trata de instrumentos imprescindibles para lograr que los alumnos se responsabilicen del trabajo que comparten en las aulas. A partir de ellos, cabe la posibilidad de organizar sesiones de análisis y discusión sobre la «marcha» del curso. Debates que han de estar bien organizados, de acuerdo con algunas de las conocidas técnicas de dinámica de grupos (Arnaiz 1987). En nuestra opinión, esta práctica ayuda a conseguir uno de los requisitos básicos del aprendizaje: la motivación, entendida como la disposición positiva hacia éste por parte del sujeto que aprende.

\section{UN POSIBLE ESQUEMA DE EVALUACIÓN DE ALUMNOS}

Seguidamente presentamos el esquema de evaluación que hemos propuesto a nuestros alumnos en los cursos anteriores. Es, de momento, un guión provisional, ya que ha de ser negociado con aquéllos, no sólo al inicio de las clases sino a lo largo de los sucesivos meses, con el fin de corregir las deficiencias que pudieran observarse en su funcionamiento. Incluye los cuatro grandes núcleos alrededor de los cuales girará la valoración de los estudiantes así como las categorías y criterios que, en cada caso, se van a contemplar.

\section{ESQUEMA DE EVALUACIÓN}

La evaluación del trabajo que se lleve a cabo en torno a la asignatura de Física y Química incluirá cuatro grandes apartados:

\section{Notas de los exámenes}

Media: Nota media de las calificaciones obtenidas en los exámenes realizados desde el comienzo del curso hasta el momento de hacer cada una de las evaluaciones.

\section{Análisis del cuaderno}

C 1: Presentación: limpieza, orden, etc.

C 2: Ausencia de errores, claridad en los conceptos, descripción de las relaciones entre unas actividades y otras, de modo que quede claro cómo se construyen los conceptos.

C 3: Exposición separada de las propias ideas -o las del grupo de trabajo- y de las resultantes de la puesta en común.

C 4: Descripción completa de las actividades experimentales, inciuyendo:

- planteamiento del problema;

- emisión, en su caso, de hipótesis; 
- diseño y planificación del experimento;

- recogida de datos y observaciones;

- interpretación de datos y establecimiento de conclusiones (enunciado, campo de aplicación, fuentes de imprecisión, etc.).

C 5: Explicación cualitativa de las expresiones matemáticas empleadas en la resolución de las actividades de aplicación -problemas, etc.-.

C Global: Media aritmética de las puntuaciones anteriores.

\section{CALIFICACIÓN;}

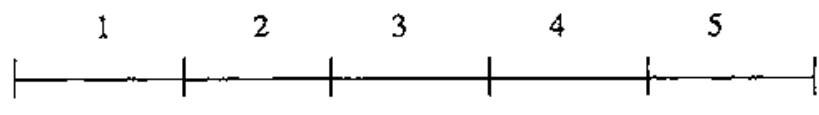

Muymal Mal Regular Bien Muybien

Tabla de observaciones (A: aula, L: laboratorio)

A 1 : Hace el trabajo propuesto en clase.

CALIFICACIÓN (para todo el grupo):

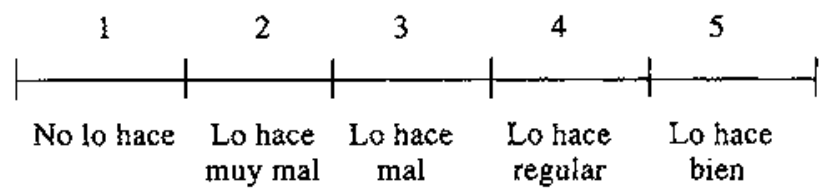

A 2 : Contesta a preguntas abiertas formuladas por el profesor o por otros compañeros.

\section{CALIFICACIÓN (individual):}

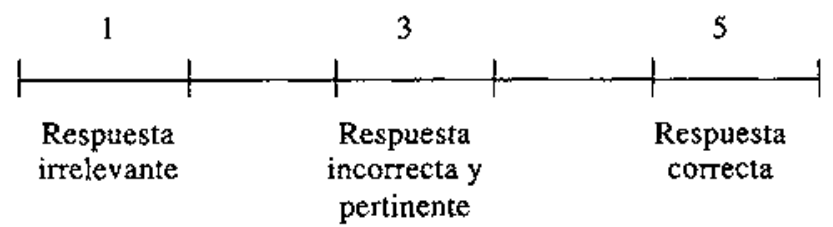

\section{A 3 : Hace preguntas significativas.}

CALIFICACIÓN (individual):

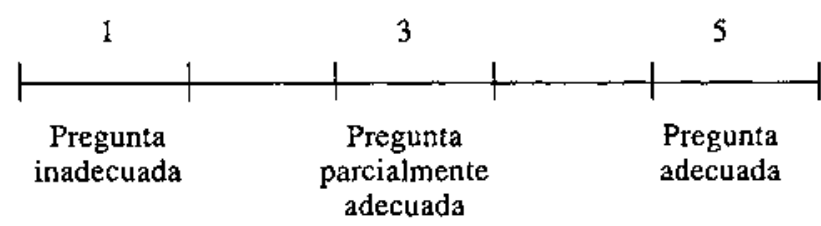

A 4: Discute y participa en el trabajo del grupo.
CALIFICACIÓN (individual):

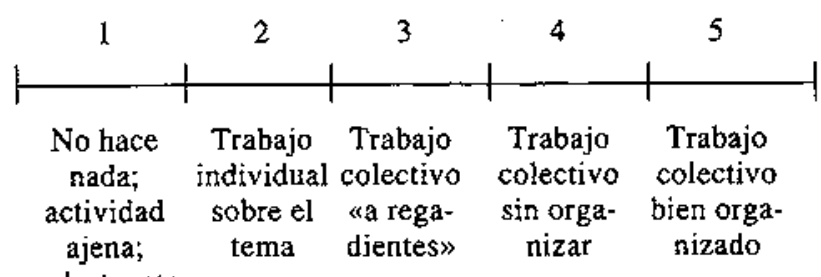

molesta; etc.

A Global: Media aritmética de las puntuaciones (A) anteriores.

L 1: Realización correcta de los pasos que constituyen el experimento completo.

\section{2: Manejo correcto de aparatos y materiales.}

L 3: Se toman precauciones para asegurar la fiabilidad de las observaciones y los resultados.

L 4: Utilización eficaz del tiempo y del espacio de trabajo disponibles.

L 5: Recogida del material y limpieza del área de trabajo.

CALIFICACIÓN (para todo el grupo):

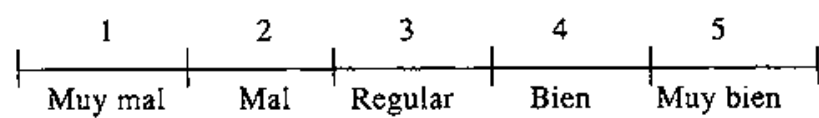

L global: Media aritmética de las puntuaciones (L) anteriores.

\section{Trabajo en casa}

Trabajo en casa: Nota media de todas las calificaciones obtenidas desde el comienzo de curso, en las actividades propuestas para hacer individualmente en casa.

\section{CALIFICACIÓN (individual):}

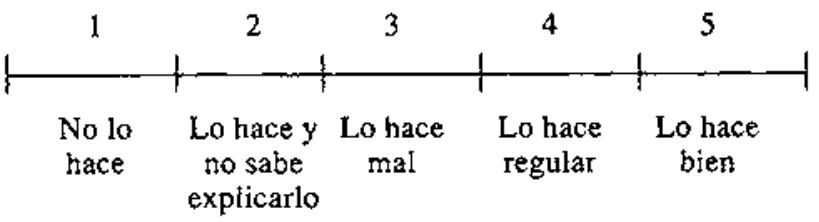

Conviene, finalmente, comentar brevemente cómo se 
aplica este esquema de evaluación en la práctica real del aula. En nuestra evaluación de los alumnos utilizamos, por razones de operatividad, números para medir cada uno de los aspectos que constituyen los cuatro núcleos del esquema. A medida que transcurre el curso, el profe. sor dispone de varias series de números, y sus correspondientes medias aritméticas, asociadas a: notas de los exámenes, análisis del cuaderno, tabla de observaciones (aula y laboratorio) y trabajo en casa. Dado que el estudiante debe percibir los avances de su propio aprendizaje, esas calificaciones deben comunicarse a los interesados por medio de un informe. No resulta difícil transcribir dichos números a un lenguaje más comprensible valiéndonos de las escalas de califícación incluidas en el esquema. El cuadro VIII muestra las calificaciones numéricas de varios alumnos de $3^{\circ}$ de BUP y los informes correspondientes.

Cuadro VIII

\begin{tabular}{lllllllllllll|}
\hline & MEDIA & C1 & C2 & C3 & C4 & C5 & A1 & A2 & A3 & A4 & L GLOBAL & TRAB. CASA \\
Aiberto & 9,5 & 4,5 & 5,0 & 4,0 & 4,5 & 4,0 & 3,5 & 3,0 & 2,0 & 4,5 & 3,7 & 4,3 \\
Carlos & 4,6 & 2,5 & 2,5 & 2,0 & 2,0 & 1,5 & 1,5 & 1,0 & 1,0 & 4,0 & 2,7 & 1,7 \\
Juana & 3,6 & 3,5 & 3,0 & 1,0 & 2,5 & 2,5 & 2,0 & 4,0 & 3,0 & 5,0 & 4,3 & 3,3 \\
\hline
\end{tabular}

\section{INFORMES}

\section{ALBERTO}

* Exámenes: Muy bien.

* Cuaderno: Muy bien en todos los aspectos.

* Aula: Podrías plantear preguntas, único aspecto que no has desarrollado.

* Laboratorio: Aunque tu actuación es aceptable, deberías mejorar en la utilización del tiempo.

* Trabajo en casa: Sigue así.

CARLOS

* Exámenes: Intenta comprender lo que se te pregunta; conceptos poco claros.

* Cuaderno: Flojo en todos los aspectos; trabájalo día a día.

* Aula: Aunque parece que participas en las discusiones de grupo, debes hacer el trabajo propuesto.

* Laboratorio: Debes mejorar en el manejo de los aparatos y en el desarollo de los experimentos.

* Trabajo en casa: No haces nada; tu verás.

JUANA

* Exámenes: Estudia más y practica el cálculo.

* Cuademo: Nomal, aunque debes distinguir entre las ideas del grupo (o las tuyas) y las resultantes de la puesta en común.

* Aula: Aunque participas en los debates en grupo, debes terminar el trabajo propuesto.

* Laboratorio: Bien en general.

* Trabajo en casa: Puedes mejorar; haz un trabajo más continuado.

No debe olvidarse, sin embargo, que la evaluación de los alumnos no es más que una de las diferentes «evaluaciones» que en una labor profesional seria habrían de tenerse en consideración.

\section{CONCLUSIONES}

Como se ha puesto de manifiesto a lo largo del artículo, el procedimiento actual de evaluación, centrado casi exclusivamente en los alumnos, sólo se preocupa de constatar resultados, olvidando la calidad del aprendizaje. Dicho procedimiento, por otro, lado, no es coherente con el modelo đe aprendizaje y enseñanza constructivista, que tanto desarrollo ha experimentado en los últimos años.

Para suplir estas deficiencias hemos recurrido a un modelo teórico ampliamente difundido en el campo de las Ciencias Sociales: el modelo etnográfico, el cual, entre otras cosas, nos ha llevado a elaborar y poner en práctica nuevas técnicas de evaluación de alumnos. Las 
estrategias de recogida de datos proporcionan además abundante información, al profesorado y al alumnado, acerca de cómo modificar su trabajo para perfeccionar la práctica cotidiana.

Con todo, debemos insistir en que se trata de una propuesta provisional, que se irá modificando a medida que

\section{REFERENCIAS BIBLIOGRÁFICAS}

ALBERTS, R.V.J., VAN BENZEKOM, P.J. y DE ROO, I., 1986. The assessment of practica! work: a choice of options, European Journal of Science Education, 3(4), pp. 361-369.

ARNÁIZ, P., 1987. Aprendizaje en grupo en el aula. (Graó: Barcelona).

CALONGHI, L., 1978. Citado en Pérez Juste.

CHRISTOFI, Ch., 1988. Assessment \& Profiling in Science. (Casell: Londres).

COLL, C., 1987. Psicologia y Curriculum. (Laia: Barcelona).

DAUVISIS, M.C., 1985. Objectifs de l'enseignement mathèmatique et docimologie. Resumen en Recherches en Didactique des Mathematiques, S(3), pp. 386-390.

DRIVER, R., 1987. Children's Learning in Science Project. (CSSME: Leeds).

ERICKSON, F., 1989. Métodos cualitativos de investigación sobre la enseñanza, en Wittrock, M.C., La investigación de la enseñanza, (Paidós MEC: Barcelona).

ESCUDERO, T., 1985. Notas sobre la evaluación científica, en Aspectos didácticos de Física y Química l. (ICE: Zaragoza).

FLANDERS, N.A., 1977. Análisis de la interacción didáctica. (Anaya: Salamanca).

FORNS, M., 1980. La evaluación del aprendizaje, en Colt, C. y Forns, M. (coords.), Áreas de intervención de Psicologia. (Horsori: Barcelona).

GIL, D., 1988. Los programas-guía de actividades: una concreción del modelo constructivista de aprendizaje de las ciencias. En Calatayud, M. L. et al, La construcción de las ciencias físicoquímicas. (Nau libres: Valencia).

GOETZ, J.P. y LECOMPTE, M.D., 1988. Etnografía y diseño cualitativo en investigación educativa. (Morata: Madrid). se obtengan nuevos resultados. Podemos adelantar que hemos observado comportamientos positivos por parte de los estudiantes, en el sentido de una mayor motivación, cuando se han dado cuenta de que, a través del método de evaluación propuesto pueden influir en la mejora de su propio aprendizaje

GUTIÉRREZ, R. et al., 1990. Enseñanza de las ciencias en la educación intermedia. (Rialp: Madrid).

HAMMERSLEY, M., 1990. Classroom ethnography. (Open University Press: Milton Keynes).

HERNÁNDEZ, F. y SANCHO, J. M., 1989. Para enseñar no basta con saber la asignatura. (Laia: Barcelona).

JIMÉNEZ, M.P., 1986. Identificación de esquemas alternativos de los alumnos/as por los profesores/as, en Actas de las $I V$ jornadas de estudio sobre investigación en la escuela. (Sevilla).

KEMPA, R., I986. Assessment in Science. (Cambridge University Press.).

KERLINGER, F.N., 1975. Investigación del comportamiento. Técnicas y metodología. (Interamericana: México).

PÉREZ JUSTE, R., 1986. Evaluación de los logros escolares. (UNED: Madrid).

PORLÁN, R., 1987. El diatio del profesor, Investigación en la Escuela, 2, pp. 77-78.

SANCHO, J. M., 1987. Entre pasillos y clases. (Sendai: Barcelona).

SANTOS, M.A., 1988. La evaluación cualitativa de planes y centros de perfeccionamiento de profesorado: una forma de mejorar la profesionalidad docente, Investigación en la Escuela, 6, pp. 21-39.

S.S.C.R., 1987.Better Science: Assessing progress. Curriculum guide 11. (Heinemann: Londres).

WALKER, R., 1989. Métodos de investigación para el profesorado. (Morata: Madrid).

WITTROCK, M.C., 1989. La investigación de la enseñanza. Métodos cualitativos y de observación. (Paidós-MEC: Barcelona). 